\title{
Robust Watermarking against Geometric Attacks using Multiple Parameter Discrete Fractional Fourier Transform and Least Significant Bit Technique
}

\author{
Deepak Sharma $^{1}$, Rajiv Saxena ${ }^{2}$ and Narendra Singh $^{3}$ \\ Department of Electronics and Communication Engineering \\ 1,3 Jaypee University of Engineering and Technology, Guna, India \\ 2 Jaypee University, Anoopshahr, India \\ ${ }^{1}$ deepakforu23@rediffmail.com,2rajiv.saxena@jiit.ac.in \\ ${ }^{3}$ narendra.singh@juet.ac.in
}

\begin{abstract}
Watermarking is an important technique used for copyright protection, authentication, tamper detection and hiding secret information in multimedia contents. In this article a new digital watermarking scheme is proposed based on multiple parameter discrete fractional Fourier transform and using least significant bit (LSB) technique. The multiple parameter discrete fractional Fourier transform (MPDFRFT) is the generalization of the discrete fractional Fourier transform (DFRFT) in terms of its different nature of its fractional orders. The MPDFRFT can converge to DFRFT when all of its fractional order parameters having a similar value. The multiple parameter discrete fractional transforms shows it's superiority over DFRFT because of that it benefitted us with an extra degree of freedom that is provided by its multiple fractional orders. The LSB technique is more preferable because of its lesser effect on the image characteristics. During the implementation of proposed scheme cover image is subdivided and MPDFRFT is applied to each subdivided image to transformed coefficients and watermark images are embedded by using LSB technique. Similarly during watermark extraction process the reverse order of MPDFRFT is applied for reconstruction of original images along with LSB extraction mechanism. The MPDFRFT fractional order parameters are used as secret key to improve the robustness of the system. The robustness of the watermark image is tested under different attacks such as additive noise, cropping, rotation and Gaussian low-pass filtering is analyzed and the results demonstrate that the embedding scheme has good performance of robustness. The imperceptibility of the proposed scheme against the hostile person is also verified by simulations results.
\end{abstract}

Keywords: Watermarking, Discrete Fractional Fourier Transform (DFRFT), Fractional Fourier Transform (FRFT), Mean square error (MSE), Multiple Parameter Discrete Fractional Fourier Transform (MPDFRFT), Least Significant bit (LSB)

\section{Introduction}

According to world wide web consortium (W3C) the growth of internet from 1995 to 2014 (March) is tremendously increased from 0.4 to 40.7 percent and subsequently the use of multimedia contents is also increased. Substantially the data manipulation, piracy, legal issues and dissemination of data kind of malicious activity invites absurd people. In this regard, watermarking technology provides an efficient solution for addressing these problems. Ultimately Watermarking is a branch of information hiding 
which is used to hide proprietary information, copyright infringement issues and authentication in digital media like photographs, digital music, or digital video [1].

On the basis of working domain watermarking schemes are broadly classified into two categories: spatial and transform domain schemes. In Spatial domain images are represented by pixels and these schemes are less complex but are not robust against attacks and low-bit carrying capacity [1-2]. On the other hand, in transform domain schemes image is segmented into multiple frequency bands and can embed more bits of watermark. These techniques are more robust and popular in comparison to spatial domain schemes [1-2]. The examples of transform domain watermarking are Discrete Fourier Transform (DFT), Discrete Cosine Transform (DCT), Discrete Wavelet Transform (DWT), singular value decomposition (SVD), Fractional Fourier Transform (FRFT) and Discrete fractional Fourier transform (DFRFT) watermarking [2-9]. Watermarks could be embedded in the spatial domain of images by modifying the pixel values or the least significant bit (LSB) values; however, more robust watermarks could be embedded in the transform domain of images by modifying the transform domain coefficients. Since robust watermarking has many applications so researchers paid more attention on transform domain watermarking which leads robust watermarking algorithms. The watermark robustness can experience against several attacks because the multimedia contents can be digitally processed. The attacks can be unintentional or unintentional. The intentional attacks are like images low pass filtering, image gamma correction and image compression while intentional attack is like cropping, salt and pepper noise. Hence the watermark has to be very robust against all these possible attacks.

Fundamental need of any healthy watermarks must have the following:

1. Transparency or fidelity

2. Robustness

3. Capacity or payload

The main applications of watermarks are:

1. Copyright protection

2. Content archiving

3. Metadata insertion

4. Broadcast monitoring

5. Tamper detection

6. Digital fingerprinting

In the mathematics literature, a generalization of the Fourier transform known as the fractional Fourier transform (FRFT), The FRFT of a signal can also be interpreted as a decomposition of the signal in terms of chirps [10]. The FRFT is also considered as a special case of the more general linear canonical transform [11], and it provides a tool to compute the mixed time and frequency components of signals. It has become a powerful and potential tool for time-varying non-stationary signal and image processing applications [12]. Due to need of its discrete version the discrete fractional Fourier transform (DFRFT) came into existence, during the development of the DFRFT, it has been considered to be a linear weighted summation of the signal and spectrum [13]. Later in 1997, the DFRFT was improved by Pei and Yeh [14]. DFRFT with discrete 
Hermite eigen vectors and an appropriate eigen value assignment rule satisfy all four important property 1) unitarity; 2) index additivity; 3) reduction to the DFT when the order is equal to unity; 4) approximation of the continuous FRFT as those of continuous FRFT [14]. Pei et.al. [16] Improved this type of discrete fractional Fourier transform (DFRFT) by modifying their eigenvectors more closely to the continuous Hermite eigenvectors. This eigen decomposition method for the DFRFT has been consolidated in [10] and applied for numerous applications as a important tool [17-18]. In 2008 Pei [19] has given a new MPDFRFT is defined from the eigen decomposition- based DFRFT by taking different fractional powers for different eigen values. The MPDFRFT is much more flexible than the DFRFT because it has order parameters while MPDFRFT is shown to have all of the desired properties for fractional transforms.

The outline of this paper is as follows: In section II the definitions of FRFT, DFRFT and MPDFRFT formulation is presented. In section III the concept LSB technique and fundamental of watermarking in DFRFT domain is discussed briefly. Section IV deals with the proposed watermarking scheme and in subsection the watermark embedding and extraction process dealt stepwise. In section $\mathrm{V}$ the performance measuring criteria on the basis of PSNR, normalized correlation coefficient (NCC) and CR are mathematically discussed. Section VI shows the experimental results of the proposed watermark scheme and discussion on results presented over existed schemes. In section VII the article is concluded with its numerical comparisons and its further future research scope.

\section{Definitions of FRFT, DFRFT and Formulation of MPDFRFT}

The FRFT is defined for $\alpha=p \pi / 2$ rotation angle of transformed signal with its $p^{\text {th }}$ transform order is given by,

$$
X_{p}(u)=F_{p}[x](u)=\int_{-\infty}^{+\infty} x(t) K_{p}(t, u) d t
$$

The FRFT operator is designated by $F_{p}$. The FRFT is periodic with period 4 .

Where the kernel is given by,

$$
K_{p}(t, u)= \begin{cases}\sqrt{(1-j \cot \alpha)} \mathrm{e}^{\mathrm{j} \pi\left(\mathrm{u}^{2} \cot \alpha+\mathrm{t}^{2} \cot \alpha-2 u t \csc \alpha\right)} & \text { for } \alpha \neq n \pi \\ \delta(t-u) & \text { for } \alpha=2 n \pi \\ \delta(t+u) & \text { for } \alpha=(2 n \pm 1) \pi\end{cases}
$$

The equation (1) can also be written as if modify $u=u / \sqrt{2 \pi}$ and $t=t / \sqrt{2 \pi}$

$$
x_{p}(u)=F_{p}[x](u)= \begin{cases}\sqrt{\frac{(1-j \cot \alpha)}{2 \pi}} \mathrm{e}^{\mathrm{j} \frac{\mathrm{u}^{2}}{2} \cot \alpha} \int_{-\infty}^{+\infty} x(t) \mathrm{e}^{j \frac{\mathrm{t}^{2}}{2} \cot \alpha-j u t \csc \alpha} & \text { for } \alpha \neq n \pi \\ x(u) & \text { for } \alpha=2 n \pi \\ x(-u) & \text { for } \alpha=(2 n \pm 1) \pi\end{cases}
$$

The $N$-point DFRFT is given by the following relationship [14, 15]

$$
F^{2 \alpha / \pi}=V D^{2 \alpha / \pi} V^{T}
$$

Where $p=2 \alpha / \pi$ the DFRFT order of the parameter and $\alpha$ is indicates the rotation angle of DFRFT. 


$$
\begin{array}{ll}
V=\left[v_{0}\left|v_{1}\right| \ldots \ldots .\left|v_{N-2}\right|\left|v_{N-1}\right|\right] & \text { For } N \text { is odd, } \\
V=\left[v_{0}\left|v_{1}\right| \ldots \ldots .\left|v_{N-2}\right|\left|v_{N-1}\right|\right] & \text { For } N \text { is even, }
\end{array}
$$

Here $v_{k}$ is the $k$-th order DFT hermite eigen vector calculated based on the [14] and [15].

$D^{2 \alpha / \pi}$ is a diagonal matrix with eigen values of DFRFT in the diagonal entries.

The $N \times N$ DFT matrix $F$ is given by,

$$
F_{k n}=\frac{1}{\sqrt{N}} e^{-j \frac{2 \pi}{N} k n} \quad 0 \leq k, n \leq N-1
$$

The computation for the DFRFT kernels for even and odd cases of $N$ is done. For the odd case the equation (4) will be converted in the following form,

$$
F^{2 \alpha / \pi}=\sum_{k=0}^{N-1} e^{-j k \alpha} v_{k} v_{k}^{T}
$$

Similarly for the even value of $N$ case the equation (4) will be converted in the form mentioned below,

$$
F^{2 \alpha / \pi}=\sum_{k=0}^{N-2} e^{-j k \alpha} v_{k} v_{k}^{T}+e^{-j N \alpha} v_{N} v_{N}^{T}
$$

The final DFRFT output is computed as a,

$$
\begin{gathered}
\left.X_{\alpha}=\sum_{k=0}^{N-1} e^{-j k \alpha} v_{k} v_{k}^{T} x \quad \text { (For the odd values of } \mathrm{N}\right) \\
X_{\alpha}=\sum_{k=0}^{N-2} e^{-j k \alpha} v_{k} v_{k}^{T} x+e^{-j N \alpha} v_{N} v_{N}^{T} x \quad \text { (For the even values of } \mathrm{N} \text { ) }
\end{gathered}
$$

If the DFRFT is further generalize on the basis of taking different fractional power for the eigen values $\lambda_{k}=\exp (-j \pi k / 2)$ of the DFT matrix. Subsequently the $N$ point $N \times N$ MPDFRFT matrix is given as,

$$
F^{\overline{2 \alpha / \pi}}=V D^{\overline{\left(2 \alpha_{1} / \pi\right)\left(2 \alpha_{2} / \pi\right)\left(2 \alpha_{3} / \pi\right) \cdots \cdots}} V^{T}
$$

The computation for the MPDFRFT kernels for even and odd cases of $N$ is done. For the odd case the equation (10) will be converted in the following form,

$$
F^{\overline{2 \alpha / \pi}}=\operatorname{V} \cdot \operatorname{diag}\left(\left(e^{-j \frac{\pi}{2} 0}\right)^{p_{0}},\left(e^{-j \frac{\pi}{2} 1}\right)^{p_{1}} \ldots \ldots \ldots \ldots . .\left(e^{-j \frac{\pi}{2}(N-1)}\right)^{p_{N-1}}\right) \cdot V^{T}
$$

For the odd values of $N$

Similarly for the even value of $N$ case the equation (10) will be converted in the form mentioned below,

$$
=V \cdot \operatorname{diag}\left(\left(e^{-j \frac{\pi}{2} 0}\right)^{p_{0}},\left(e^{-j \frac{\pi}{2} 1}\right)^{p_{1}} \ldots \ldots\left(e^{-j \frac{\pi}{2}(N-2)}\right)^{p_{N-2}}\left(e^{-j \frac{\pi}{2}(N)}\right)^{p_{N}}\right) \cdot V^{T}
$$

For the even values of $N$ When $\operatorname{diag}\left(u_{1}, u_{2}, \ldots . ., u_{n}\right)$ represents the $N \times N$ diagonal matrix whose diagonal elements are $u_{1}, u_{2}, \ldots \ldots, u_{n}$. In equation (11) and (12) $\bar{p}$ is a $1 \times N$ parameter vector consisting of the $N$ independent order parameters of the MPDFRFT, 


$$
\bar{p}=\left\{\begin{array}{lc}
\left(p_{0}, p_{1}, p_{2}, \ldots \ldots ., p_{N-1}\right) & \text { for } \mathrm{N} \text { odd } \\
\left(p_{0}, p_{1}, p_{2}, \ldots \ldots \ldots, p_{N-2}, p_{N}\right) & \text { for } \mathrm{N} \text { even }
\end{array}\right.
$$

The MPDFRFT of $X_{\bar{p}}$ of the $N \times 1$ data vector $\mathrm{x}$ with the parameter vector $\bar{p}$ computed as,

$$
X_{\bar{p}}=F^{\overline{2 \alpha / \pi}} \mathrm{X}
$$

\section{LSB Technique and Concept of Watermarking in DFRFT Domain}

The least significant bit (LSB) technique is one of the oldest techniques for embedding the secret information inside a cover image of pixel values without perceptible distortions. The LSB technique replaces the least bits (one, two or could be more) of pixels of cover image by bits of the secret image because to our human eye, changes in the value of the LSB are imperceptible. Generally 1 to 4 least bits of cover image are used to embed the private information. After embedding the secret information like watermarks, like text, message, images, and logos etc into cover image, generates watermarked image which resemble an original image and does not changes the quality of perception of cover image. Usually only half of bits of cover image are required to conceal a secret message. Moreover, bit insertion of dual watermarks into the cover image is followed in such a way. In a gray scale image, each pixel has pixel value range from 0 to 255 . So the binary representation of each pixel consist 8-bits. The watermark location in cover image consist each pixel of both the watermark and embedded in cover image form watermarked image which is full of two pixel values of watermark mono and cover image. The figure 1 shows the LSB insertion mechanism in its block level representation.

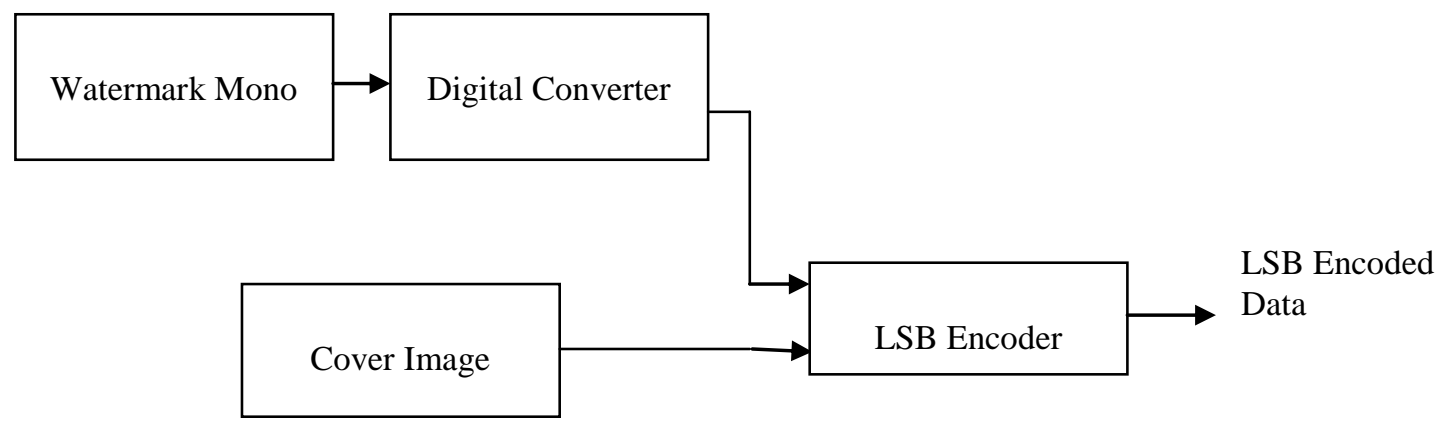

Figure 1. LSB Insertion Mechanism

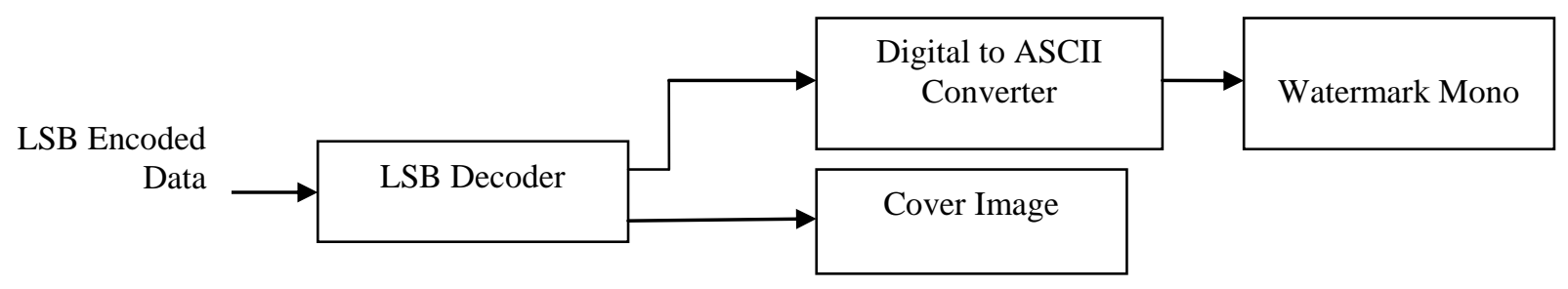

Figure 2. LSB Extraction Mechanisms

In Figure 2 the subsequent reverse process of LSB decoding mechanism is shown which is applied at the receiver side. 
The concept of watermarking in DFRFT domain lies on the fact that the most of the energies of image signal centralize on visual important component; the ability of resistance of watermark becomes stronger after being embedded on this sector, because compression and the filtering both keep the key components of the image signal. Mathematically in DFRFT domain, if the digital watermark were embedded in the highest coefficients, it could produce image deformation, while if it were embedded in the lowest coefficients, it could be cleaned by image compression or filtering. Here we choose to embed the watermark signal into the visual sub-important component of the image, which not only avoid image distortion but also provides a better robustness with the help of MPDFRFT, to make a compromise between perceptibility and robustness.

\section{Proposed Watermarking Scheme}

In this section, the proposed method described in two subsections where first section reveals the watermark insertion or embedding procedure at the transmitter side while second subsection explain the procedure of watermark detection or extraction at the receiver side stepwise.

\section{A. Watermark Embedding Procedure as follows:}

Step 1: The cover image in which watermarks are embedded is, firstly, divided into the window size of $8 \times 8$.

Step 2: Perform 2D-MPDFRFT to each window with parameter vector $p$ and $q$ in the range of 0 to 4 .

Step 3: Select 8 pixels form each transformed window randomly and then covert them into binary form. If the transformed value is negative, consider it as positive value and then convert it into binary form.

Step 4: Convert all pixels of watermark into the 8-bit binary form and these watermarks are embedded in the leftmost part of the $8 \times 8$ window of cover image in which the selected pixels for embedding, Now this one bits from watermark is embedded in the one LSB positions of the transform coefficient of the selected pixels of the cover image.

Step 5: Reversely, convert the modified binary value of selected pixels into decimal form.

Step 6: Perform the 2D-MPDFRFT with its reverse parameter vector $(-p,-q)$.

Step 7: Repeat step 1 to 6 until all windows of cover image are completed and finally merge all these window of size $8 \times 8$.

\section{B. Watermark Extraction Process is formulated as follows:}

Step 1: Split the watermarked image into the window size of $8 \times 8$.

Step2: Perform 2D-MPDFRFT on each window with the same transform order and Parameter vectors with $(p, q)$ in the range of 0 to 4 . 
Step 3: Select the same random pixels, as taken during embedding at transmitter side, from the each window.

Step 4: Convert those pixels into 8-bit binary form and extract the LSB bit and filled with zero bits results there are fewer changes in its decimal value.

Step 5: Combine all bits of first LSB positions extracted from the selected pixels and convert into decimal form to produce the pixels of watermark image and convert into decimal form.

Step 6: Repeat steps from 2 to 5 until all the pixels of watermark are produced.

\section{Performance Measuring Criterion}

In this section, the robustness and security of the proposed algorithm is performed based on parameter peak signal to noise ratio, normalized correlation coefficient and compression ratio. A 512 x 5128 bit gray-scale images "Lena" and a 64 x 64 watermark binary image are chosen to demonstrate the performance. From literature review, it is well known that there is a tradeoff between imperceptibility and robustness in watermarked image. A good watermarking approach should retain a good image quality and should provide a strong robustness. Hench the peak signal-to-noise ratio (PSNR) and Normalized correlation coefficient (NCC) are calculated for the evaluation of the imperceptibility and robustness of the watermarking algorithm.

\section{1) Peak Signal to Noise Ratio (PSNR):}

The imperceptibility of the watermark can be calculated by calculating the PSNR and it is defined in terms of the mean square errors (MSE). The MSE value is measured by taking the difference between a watermark image $W(i, j)$ and its original image $L(i, j)$. The typical values of PSNR lie between $30 \mathrm{~dB}$ to $40 \mathrm{~dB}$.

$$
\begin{gathered}
\text { Peak Signd to Noise Ratio }(P S N R)=10 \log _{10}\left[\frac{255 \times 255}{M S E}\right] d B=10 \log _{10}\left[\frac{M A X_{I}^{2}}{M S E}\right] d B \\
\text { (For gray scale) } \\
\text { Mean square Error(MSE) }=\frac{1}{R \times S} \sum_{i=1}^{R} \sum_{j=1}^{S}[L(i, j)-W(i, j)]^{2}
\end{gathered}
$$

Where $R$ and $S$ indicates the size of the image.

If the PSNR of watermarked image is greater than $30 \mathrm{~dB}$ then it is very difficult to identify the difference between cover image and watermarked image. The invisibility of watermark is good quality.

\section{2) Normalized Correlation Coefficient (NCC):}

The NCC is calculated between the watermark image (W) and extracted watermark image (W').

$$
\mathrm{NCC}=\frac{\sum_{\mathrm{i}=1}^{\mathrm{R}} \sum_{\mathrm{j}=1}^{\mathrm{S}} W(i, j) W^{\prime}(i, j)}{\sqrt{\sum_{\mathrm{i}=1}^{\mathrm{R}} \sum_{\mathrm{j}=1}^{\mathrm{S}}\left[W^{\prime}(i, j)\right]^{2}} \sqrt{\sum_{\mathrm{i}=1}^{\mathrm{R}} \sum_{\mathrm{j}=1}^{\mathrm{S}}[W(i, j)]^{2}}}
$$


The range of normalized correlation coefficient lies between 0 to1. The NCC is unity when received watermark image is exactly same as extracted watermark image.

\section{3) Compression Ratio (CR)}

It is defined as the ratio of size compressed image to the size of original image and is given below,

$$
\mathrm{CR}=\frac{\text { Size of Compressed Image }}{\text { Size of Original Image }} \times 100 \quad(\text { In percentage })
$$

\section{Simulation Results and Discussion}

In this segment, the numerical simulations for proposed watermark scheme for embedding and extraction are shown. The simulation is performed on Lena gray test image of sizes $512 \times 512$ with a watermark logo of JUET with size $64 \times 64$ at the parameter vector $p, q$ at the value of 0.65 and .8 respectively for optimum results. In the next subsection of same segment the investigation is done against the different types of the intentional and unintentional attacks like JPEG compression, salt and pepper noise, Gaussian noise, Gaussian low pass filtering, cropping, rotation and median filtering operation performed on watermarked image and then watermark extracted under these operations.

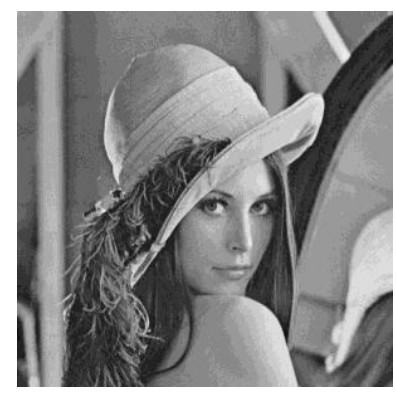

Figure 3. (a) Lena Cover Image

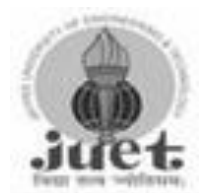

(b) Watermark Image

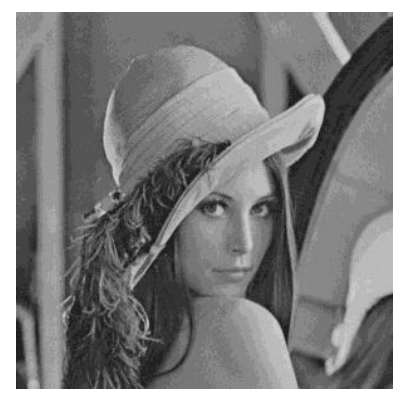

(c) Watermarked Image

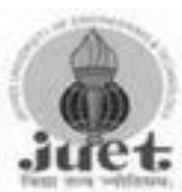

(d) Extracted watermark

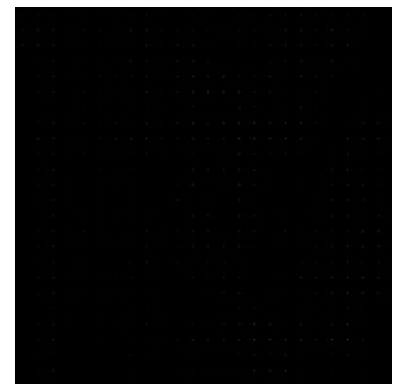

(e) Difference between cover and watermarked image

Figure 3 (a) shows the original cover image of "Lena" test image with size 512 x 512 and 3(b) shows the watermark logo of "juet" of size 64 x 64 which is to be hide under cover image of Lena. After watermark algorithm implementation figure 3(c) is produced as a watermarked image having a high perceptible quality with PSNR 64.2dB after extraction algorithm the watermark is extracted shown in figure 3 (d). The figure 3(d) shows the difference between cover image and watermarked image that provides almost nil information about the watermark insertion information. 


\section{A. JPEG compression}

Table 1 shows the extracted results from a JPEG compressed version of the watermarked images with different compression ratios on the basis of JPEG quality factor. The quality of watermarked images is still in a good situation, even under a high compression ratio is selected. Experimental results show for the quality factor selected 80, 70, 60, 50, and 40 at that the proposed watermarking scheme has more recognizable information of copyright ownership and get watermark little faded after $Q=50$ but still the quality of watermarked image is having good perceptibility and shows robust against JPEG compression attacks.

Table 1. Watermarked and extracted watermark Images at different CR

\begin{tabular}{|c|c|c|}
\hline $\begin{array}{c}\text { JPEG Quality } \\
\text { Factor }\end{array}$ & Watermarked Image & Extracted Watermark \\
\hline 70 & & \\
\hline 60 & & \\
\hline & & \\
\hline
\end{tabular}




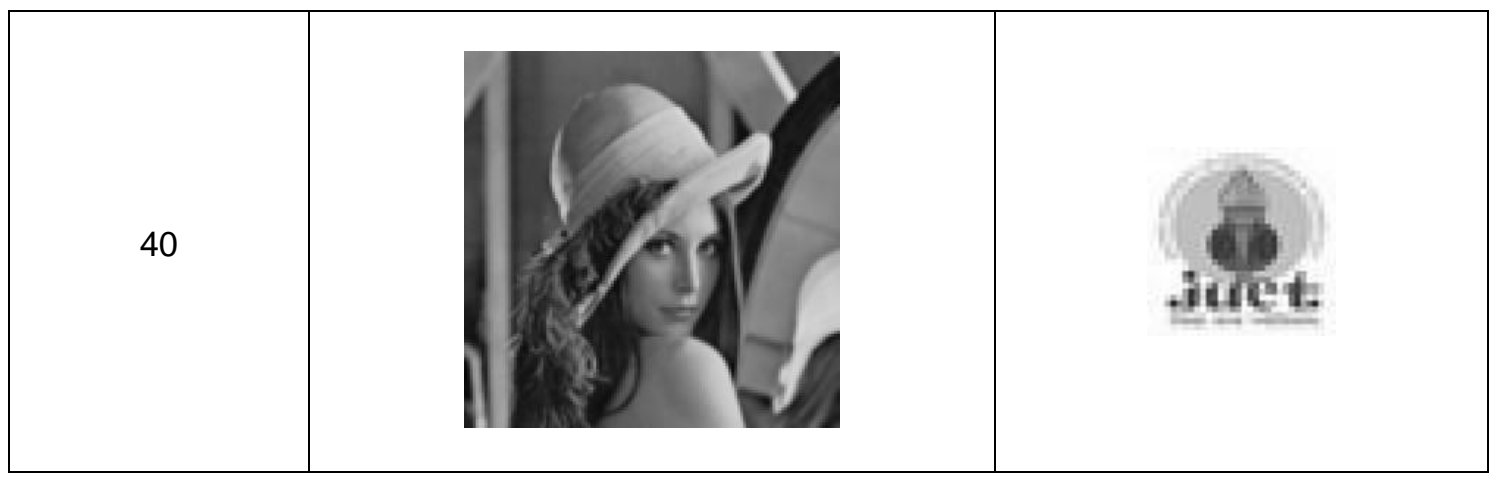

In table 2 the performance is shown in a tabular form with comparison of already similar existing schemes and from that it can be seen clearly that the proposed scheme shows better normalized correlation coefficient along with higher perceptibility of in terms of PSNR.

Table 2. JPEG compression and watermark extraction

\begin{tabular}{|c|c|c|c|c|c|c|}
\hline Schemes & JPEG Quality Factor & 80 & 70 & 60 & 50 & 40 \\
\hline \multirow{2}{*}{$\begin{array}{c}\text { Sun et.al } \\
{[20]}\end{array}$} & $\mathrm{NC}$ & --- & 0.9970 & 0.9920 & 0.9618 & 0.7505 \\
\cline { 2 - 7 } & PSNR (in dB) & --- & $31.35 \mathrm{~dB}$ & $30.92 \mathrm{~dB}$ & $30.87 \mathrm{~dB}$ & $29.41 \mathrm{~dB}$ \\
\hline $\begin{array}{c}\text { Lang et. } \\
\text { al. [21] }\end{array}$ & $\mathrm{NC}$ & --- & .9995 & 0.9920 & 0.9173 & 0.8770 \\
\hline \multirow{2}{*}{\begin{tabular}{c} 
Proposed \\
\cline { 2 - 7 }
\end{tabular}} & $\mathrm{NC}$ & 0.9996 & 0.9990 & 0.9980 & 0.9940 & 0.9891 \\
\hline \multirow{2}{*}{$\begin{array}{c}\text { Navas et. } \\
\text { al. [22] }\end{array}$} & PSNR (in dB) & $58.81 \mathrm{~dB}$ & $57.20 \mathrm{~dB}$ & $53.40 \mathrm{~dB}$ & $48.31 \mathrm{~dB}$ & $31.61 \mathrm{~dB}$ \\
\cline { 2 - 7 } & $\mathrm{NC}$ & 0.9991 & 0.9982 & 0.9940 & 0.9834 & 0.8015 \\
\hline
\end{tabular}

The figure 4 shows the variations of PSNR versus quality factor for proposed scheme and the schemes given in reference [20] and [22]. 


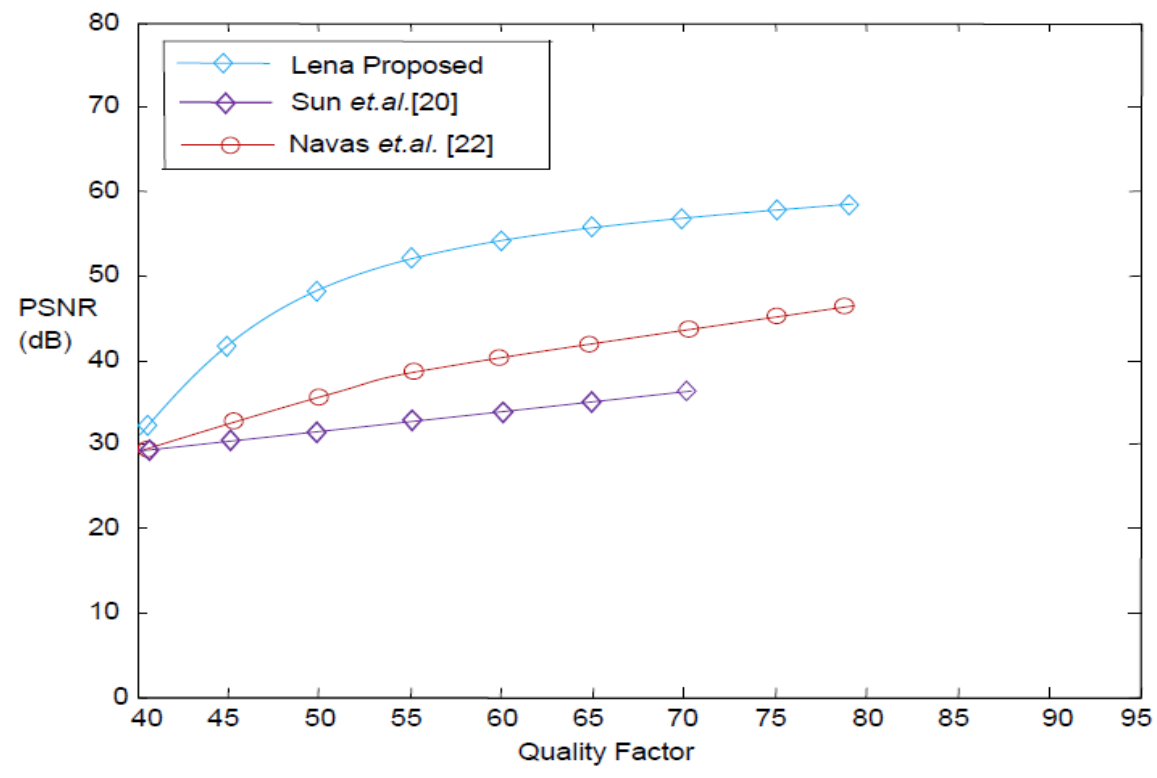

Figure 4.Variation of Quality factor versus PSNR

\section{B. Salt and Pepper Noise}

Pepper-Salt noise causes due to on and off pixels. The figure 5 (a) shows a corrupted watermarked image by a salt and pepper noise at the density of 0.12 while in figure 5 (b) shows the extracted watermark. The watermarked image is still recognizable at the higher density of salt and pepper noise level. In Table 3 shows extractive watermarks and their NCC after the watermarked Lena image adding Salt and Pepper Noise by gradually increasing the density of salt and pepper noise. The table also shows a comparative analysis of proposed scheme with the similar existed scheme based on DFRFT, DWT and hyper chaos based watermarking scheme.

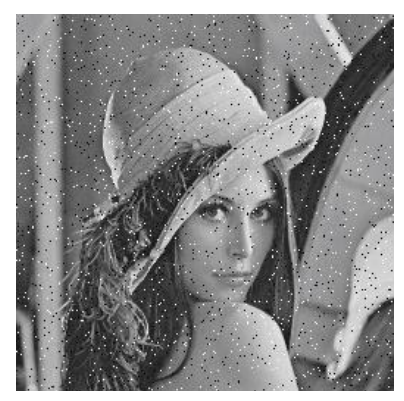

Figure 5. (a) Watermarked Image corrupted by Salt and Pepper noise

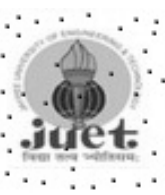

(b) Extracted watermark image 
Table 3. Salt and Pepper Noise Watermark Extraction

\begin{tabular}{|c|c|c|c|c|c|c|c|c|}
\hline \multirow{2}{*}{ Schemes } & $\begin{array}{c}\text { Salt and } \\
\text { Pepper } \\
\text { noise } \\
\text { density }\end{array}$ & 0.01 & 0.03 & 0.05 & 0.06 & 0.07 & 0.09 & 0.12 \\
\hline \multirow{2}{*}{$\begin{array}{c}\text { Sun et.al. } \\
\text { (2012) [20] }\end{array}$} & $\mathrm{NCC}$ & 0.9981 & 0.9931 & 0.9783 & --- & 0.9546 & ---- & ---- \\
\cline { 2 - 9 } & $\begin{array}{c}\text { PSNR (in } \\
\mathrm{dB} \text { ) }\end{array}$ & $25.82 \mathrm{~dB}$ & $21.91 \mathrm{~dB}$ & $19.97 \mathrm{~dB}$ & --- & $18.57 \mathrm{~dB}$ & ---- & ---- \\
\hline $\begin{array}{c}\text { Lang et.al. } \\
(2012)[21]\end{array}$ & $\mathrm{NCC}$ & ----- & 0.9959 & ---- & 0.9649 & ---- & 0.9304 & 0.8884 \\
\hline \multirow{2}{*}{\begin{tabular}{c} 
Proposed \\
\cline { 2 - 9 }
\end{tabular}} & $\mathrm{NCC}$ & 0.9995 & 0.9986 & 0.9980 & 0.9876 & 0.9820 & 0.9720 & 0.9280 \\
\hline $\begin{array}{c}\text { Navas et.al. } \\
\text { (2008) [22] }\end{array}$ & $\mathrm{NCC}$ (in & $40.35 \mathrm{~dB}$ & $30.92 \mathrm{~dB}$ & $26.20 \mathrm{~dB}$ & $25.41 \mathrm{~dB}$ & $24.95 \mathrm{~dB}$ & $23.87 \mathrm{~dB}$ & $19.92 \mathrm{~dB}$ \\
\hline
\end{tabular}

Figure 6 shows the variation of the salt and Pepper noise density versus PSNR for proposed scheme and schemes shown in reference [20] and [22].

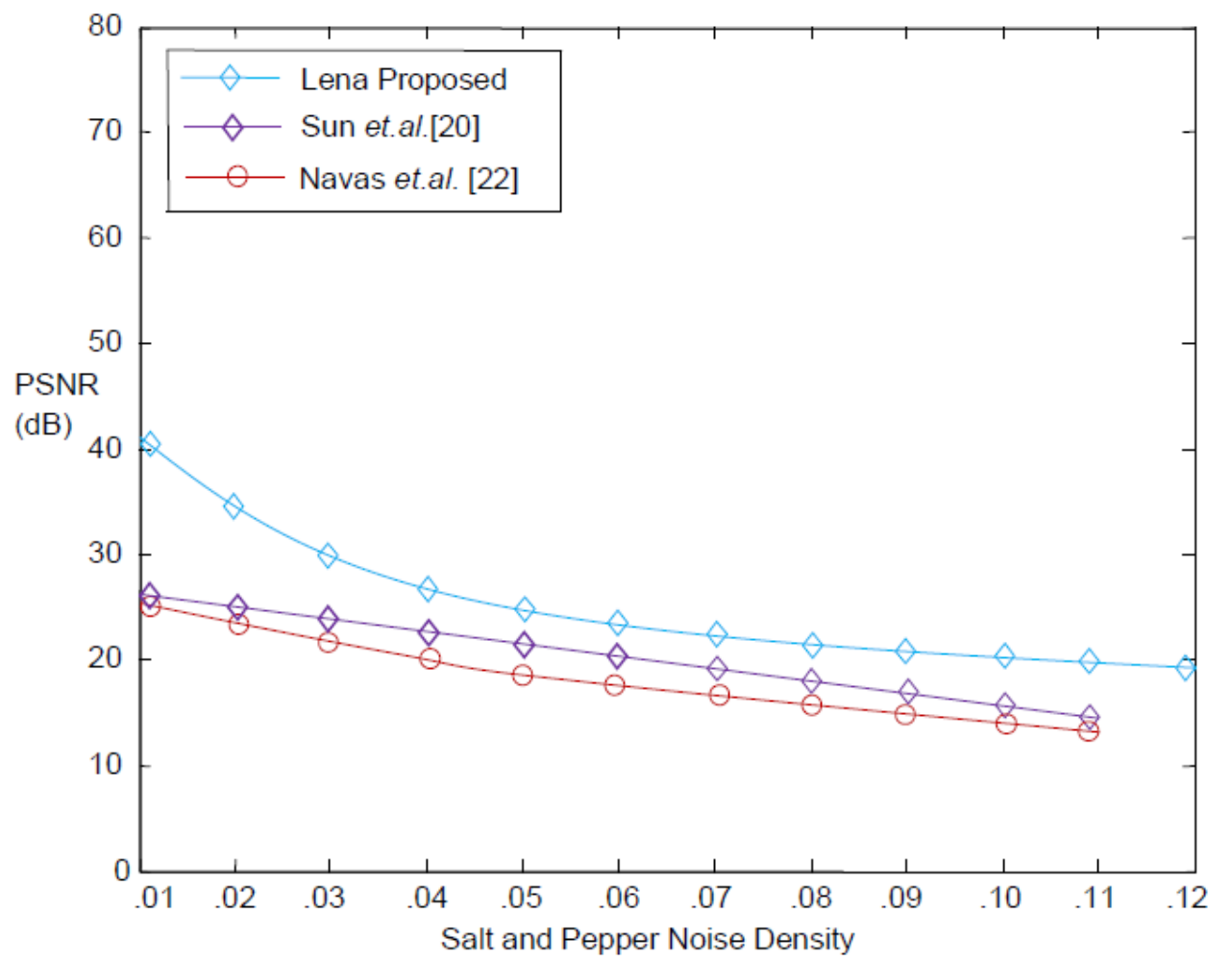

Figure 6. Variation of PSNR versus Salt and Pepper Noise Density Level

\section{Gaussian noise}

Similarly, here another kind of attack against AWGN attack is tested for proposed scheme in comparative manner with schemes provided in [20], [21] and [22]. 


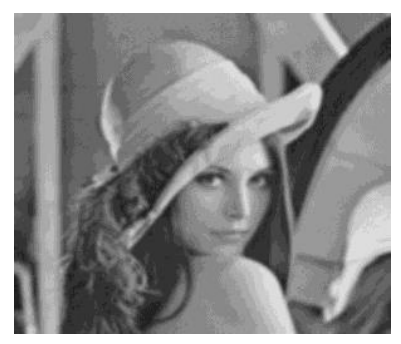

Figure 7. (a) Watermarked Image Corrupted by Gaussian Noise

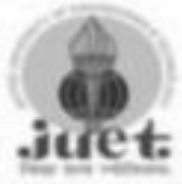

(b) Extracted Watermark Image

Table 4. Gaussian Noise Watermark Extraction

\begin{tabular}{|c|c|c|c|c|c|}
\hline Schemes & $\begin{array}{c}\text { Gaussian noise density with } \\
\text { zero mean }\end{array}$ & 0.01 & 0.03 & 0.05 & 0.07 \\
\hline $\begin{array}{c}\text { Sun et.al. (2012) } \\
\text { [20] }\end{array}$ & NCC & 0.9943 & 0.9539 & 0.8704 & 0.8684 \\
\hline $\begin{array}{c}\text { Lang et.al. (2012) } \\
\text { [21] }\end{array}$ & NCC & ---- & 0.9250 & ---- & ---- \\
\hline Proposed & NCC & 0.9970 & 0.9760 & 0.9684 & 0.9268 \\
\hline $\begin{array}{c}\text { Navas et.al. } \\
\text { (2008) [22] }\end{array}$ & NCC & Low & Low & Low & Low \\
\hline
\end{tabular}

\section{Gaussian low-pass filter}

The scheme also shows some improvement when the corrupted watermark image is passed through a Gaussian low pass filtering operation. The results are shown in Figure 8 (a) for watermarked image passed through Gaussian filter and respective watermark is extracted in Figure 8(b).

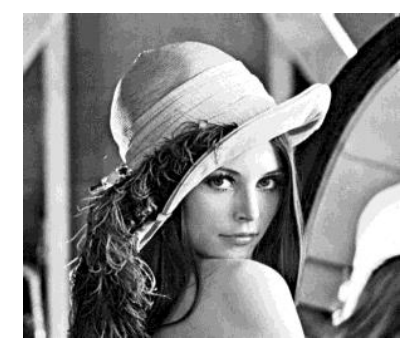

Figure 8. (a) Watermarked Image Passed through Gaussian Filter

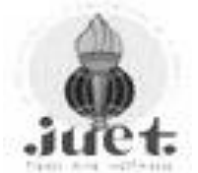

(b) Extracted watermark image 


\section{E. The cropping}

In Figure 9 (a), (c), and (e) shows the cropped image at the level of $1 / 4,1 / 2$ for upper and lower image size respectively and in Figure (b), (d) and (f) the extracted watermark images are shown respectively with small deterioration.

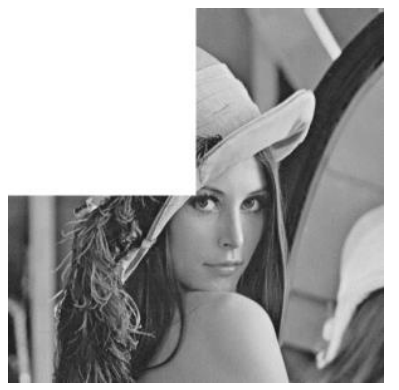

Figure 9. (a) Cropped by $1 / 4$ (b) Extracted watermark (c) Cropped by $1 / 2$ Upper Section
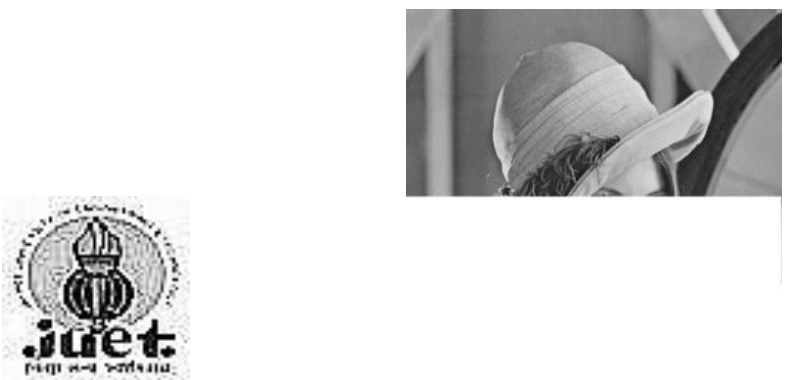

(d) Extracted Watermark

(e)

(e) Cropped by
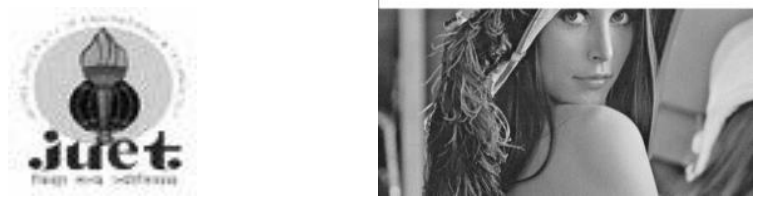

\section{F. Rotation}

Figure 10 shows the robustness of the watermarked image against the rotation effect. The extracted watermark is distorted when watermark image is distorted by an amount of rotation of $180^{\circ}$ while it shows better resistance at the rotation of $90^{\circ}$.

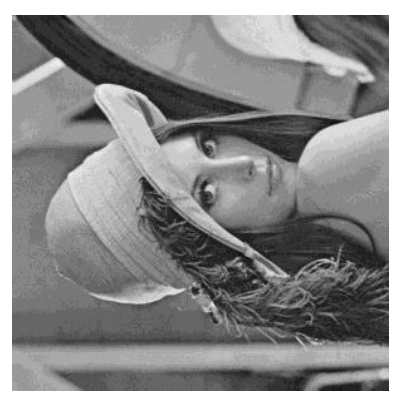

Figure 10 (a) Rotated by $90^{\circ}$

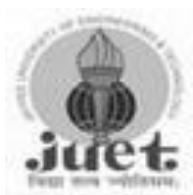

(b) Extracted Watermark

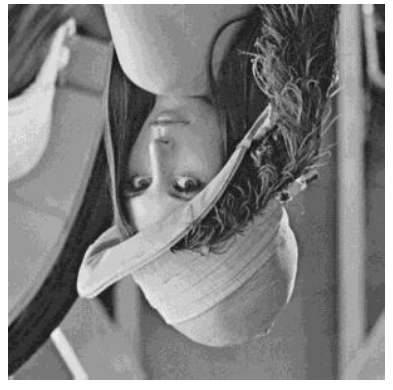

(c) Rotated by $180^{\circ}$ 


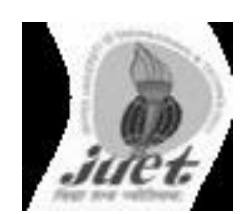

(d) Extracted watermark

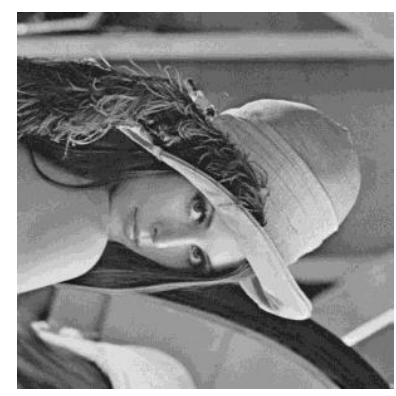

(e) Rotated by $-90^{\circ}$

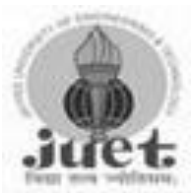

(f) Extracted Watermark

\section{G. The Median Filtering}

The distorted results can also improved by applying median filtering as shown in figure 11 (a), (b) and (c).
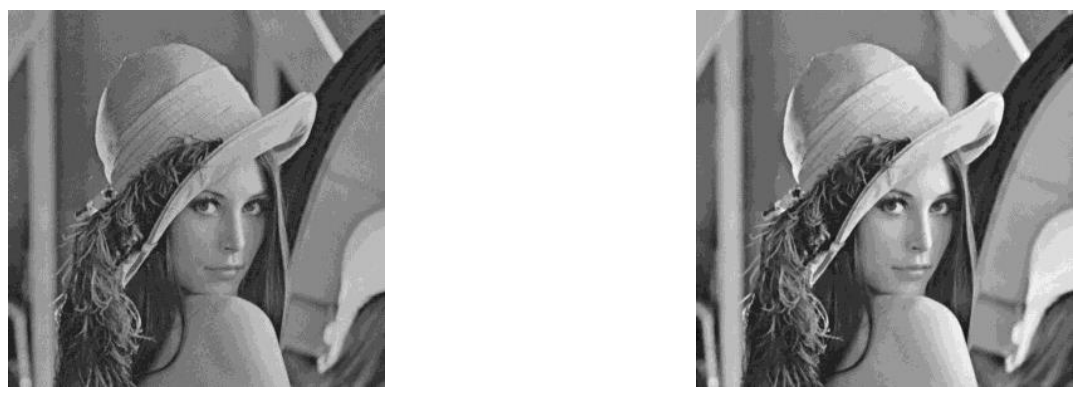

Figure 11.(a) Watermarked Image (b) Watermarked after applying Median Filtering

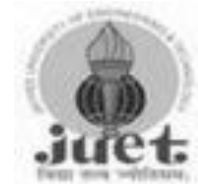

(c) Extracted Watermark in Median Filtering

\section{Conclusion}

This article discusses a watermarking scheme based on multiple parameter discrete fractional Fourier transform with LSB technique The proposed scheme shows $34.74 \mathrm{~dB}$ improvement in PSNR by the scheme proposed in [21] by Lang, 32.17dB by [20] in Sun et.al., and $0.9 \mathrm{~dB}$ improvement in PSNR by the scheme given by Navas in [22]. The scheme shows better robustness due to the multiple parameter applicability in the discrete fractional Fourier transform. The quality of the watermarked image is good both visually and in terms of PSNR. Finally, the proposed watermarking method is robust to typical intentional and unintentional attack like JPEG compression, Cropping, rotation, AWGN, Salt and Pepper noise and shows good response towards the filtering operation. Further the proposed scheme may also incorporate the steganography and encryption application in future. 


\section{Acknowledgements}

The authors thankfully acknowledge all the authorities of Jaypee University of Engineering \& Technology, Guna (M.P.), PIN-473226, India.

\section{References}

[1] L. I. Cox, Miller and Bloom, "Digital watermarking", Morgan Kaufmann Publisher, San Francisco, CA, USA, (2002).

[2] C. Yaw Low, AB. Jin Toeh and C. Tee, "Fusion of LSB and DWT Biometric Watermarking for Offline Handwritten Signature", Proceedings of Congress on Image and Signal Processing, CISP'08, Vol. 5, (2008), pp. 702-708.

[3] C. T. Hsu and J. L. Wu, "The Hidden Digital Watermarks in Images", IEEE Trans. on Image Processing, vol. 8, no. 1 , (1999), pp. 58-68.

[4] V. Solachidis and I. Pitas, "Circularly Symmetric Watermark Embedding in 2D-DFT domain", IEEE Trans. on Image Processing, vol. 10, no. 11 (2001) pp. 1741-1753.

[5] I. J. Cox and M. L. Miller, "The First 50 Years of Electronic Watermarking", Eurasip Journal of Applied Signal Processing, vol. 2, no. 1, (2002), pp. 126-132.

[6] M. D. Swanson, B. Zhu, and A. H. Tewfik, "Transparent robust image watermarking", Proceeding of ICIP, (1996), pp. 211-214.

[7] J.R. Hernndez, M. Amado, F. P. Gonzlez, "DCT-domain watermarking techniques for still images detector performance analysis and a new structure", IEEE Transactions on Image Processing, vol. 9, no. 1, (2000), pp. $55-68$.

[8] I. Djurovic, S. Stankovic and I.Pitas, "Digital watermarking in the fractional Fourier transformation domain", Journal of Network and Computer Applications, vol. 24, (2001), pp. 167-173.

[9] N. M. Makbol and Bee Ee Khoo, "Robust blind image watermarking scheme based on Redundant Discrete Wavelet Transform and Singular Value Decomposition", International Journal of Electronics and Communications, (AEÜ), vol. 67, (2013), pp. 102-112.

[10] V. Namias, "The fractional order Fourier transform and its application to quantum mechanics," J. Inst. Math. Appl., vol. 25, (1980), pp. 241-265.

[11] K. B. Wolf, "Construction and properties of canonical transform", in Integral Transforms in Science and Engineering. New York: Gordon and Breech, (1979), ch. 9.

[12] L. B. Almeida, "The fractional Fourier transform and time-frequency representations," IEEE Trans. Signal Processing, vol. 42, (1994), pp. 3084-3091.

[13] B. Santhanam and J. H. McClellan, "The discrete rotational Fourier transform," IEEE Trans. Signal Processing., vol. 42, (1996), pp. 994-998.

[14] S. C. Pei and M. H. Yeh, "Improved discrete fractional Fourier transform," Opt. Lett., vol. 22, no. 14, (1997), pp. 1047-1049.

[15] S. C. Pei, M. H. Yeh, and C. C. Tseng, "Discrete fractional Fourier transform based on orthogonal projections," IEEE Trans. Signal Processing, vol. 47, (1999), pp. 1335-1348.

[16] C. Candan, M. A. Kutay and H. M. Ozaktas, "The discrete fractional Fourier transform", IEEE Trans. on Signal Process., vol. 48, no. 5, (2000), pp. 1329-1337.

[17] S. C. Pei and M. H.Yeh, "Discrete fractional Hilbert transform," in Proc. IEEE Int. Symp. Circuits Syst., (1998), pp. 506-509.

[18] S. C. Pei, C. C. Tseng, M. H. Yeh, and J. J. Shyu, "Discrete fractional Hartley and Fourier transforms," IEEE Trans. Circuit Syst. II, vol. 45, (1998), pp. 665-675.

[19] S. C. Pei and W. L. Hsue, "The Multiple-Parameter Discrete Fractional FourierTransform", IEEE Signal Processing Letters, vol. 13, no. 6, (2006), pp. 329-332.

[20] Jin-ying Sun, Jun Lang, Chengqiang Miao, Nan Yang and Shenquan Wang, "A Digital Watermarking Algorithm based on Hyperchaos and Discrete Fractional Fourier Transform", 5th International Congress on Image and Signal Processing (CISP), (2012), pp. 553-556

[21] Jun Lang, Jin-ying Sun,Wen-fang Yang, “A Digital Watermarking Algorithm Based on Discrete Fractional Fourier Transformation”, 2012 International Conference on Computer Science and Service System (2012), pp. 692-694 
[22] K. A. Navas, Ajay, M. C . Lekshmi, M., T. S. Archana, M. Sasikumar, "DWTDCT-SVD Based Watermarking" $3^{\text {rd }}$ International Conference on Communication Systems Software and Middleware and Workshops, (2008), pp. 271 - 274.

\section{Authors}

Deepak Sharma, Obtained his M. Tech. (Microwave Engineering) from Madhav Institute of Technology and Science (MITS), Gwalior (M.P.) in 2006. Currently working as an Assistant Professor in Jaypee University of Engineering and Technology (JUET), Guna Before joining JUET, he worked as a Lecturer in Electronics Department, MITS, Gwalior (M.P.). Presently, he is pursuing his Ph.D. degree from Jaypee University of Engineering and Technology, Guna under the guidance of Prof. Rajiv Saxena and Dr. N. Singh. His Research areas includes Digital Signal processing, Image processing and Integral Transforms.

Rajiv Saxena, born at Gwalior in Madhya Pradesh in 1961, obtained B.E. (Electronics \& Telecommunication Engineering) in the year 1982 from Jabalpur University, Jabalpur. Subsequently, Dr. Saxena joined the Reliance Industries, Ahmedabad, as Graduate Trainee. In 1984, Dr. Saxena joined Madhav Institute of Technology \& Science, Gwalior as Lecturer in Electronics Engineering. He obtained his M.E. (Digital Techniques \& Data Processing) from Jiwaji University, Gwalior in 1990. The Ph. D. degree was conferred on him in 1996-97 in Electronics \& Computer Engineering from IIT, Roorkee (erstwhile UOR, Roorkee). Dr. Saxena was former head and professor at Jaypee University, Guna (M.P.) for seven year. Currently Dr. Saxena is Director at Jaypee University, Anoopshahr (U.P.).

Narendra Singh, born at Orai in Uttar Pradesh in 1977, obtained B. Tech. (Electronics \& Instrumentation Engineering) in the year 2001 from Bundelkhand University, Jhansi, M. Tech. in Digital Systems in the year 2004 from M.N.N.I.T. Allahabad (Deemed University) and obtained PhD from Jaypee University of Engineering \& Technology, Guna, (M.P.) in the year 2012. Narendra Singh joined JIET, Guna, as lecturer in Electronics and Communication Engineering in 2004. Currently he is assistant professor (senior grade) in ECE department at JUET, Guna. 
International Journal of Security and Its Applications Vol.8, No.5 (2014) 